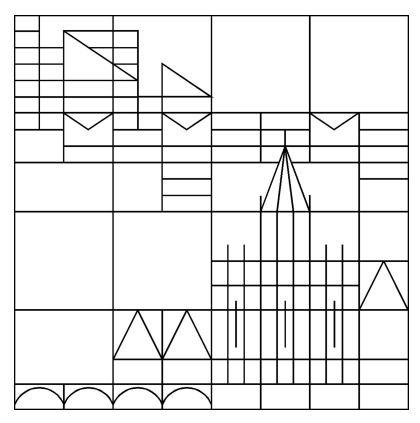

\title{
Compressible Navier-Stokes equations with hyperbolic heat conduction
}

\author{
Yuxi $\mathrm{Hu}$ \\ Reinhard Racke
}

Konstanzer Schriften in Mathematik

Nr. 332, November 2014

ISSN 1430-3558 



\title{
COMPRESSIBLE NAVIER-STOKES EQUATIONS WITH HYPERBOLIC HEAT CONDUCTION
}

\author{
YUXI HU AND REINHARD RACKE
}

\begin{abstract}
In this paper, we investigate the system of compressible Navier-Stokes equations with hyperbolic heat conduction, i.e., replacing the Fourier's law by Cattaneo's law. First, by using Kawashima's condition on general hyperbolic parabolic systems, we show that for small relaxation time $\tau$, global smooth solution exists for small initial data. Moreover, as $\tau$ goes to zero, we obtain the uniform convergence of solutions of the relaxed system to that of the classical compressible Navier-Stokes equations.
\end{abstract}

Keywords: Compressible Navier-Stokes; hyperbolic heat conduction; global solution; singular limit

AMS classification code: $35 \mathrm{~B} 25,76 \mathrm{~N} 10$

\section{INTRODUCTION}

The compressible Navier-Stokes equations with heat conducting in $\mathbb{R}^{n} \times[0,+\infty)$ $(n \geq 1)$ can be written in the following form

$$
\left\{\begin{array}{l}
\partial_{t} \rho+\operatorname{div}(\rho u)=0, \\
\partial_{t}(\rho u)+\operatorname{div}(\rho u \otimes u)+\nabla p=\operatorname{div} S, \\
\partial_{t}\left(\rho\left(e+\frac{1}{2} u^{2}\right)\right)+\operatorname{div}\left(\rho u\left(e+\frac{1}{2} u^{2}\right)+u p\right)+\operatorname{div} q=\operatorname{div}(u S),
\end{array}\right.
$$

where $\rho, u=\left(u_{1}, u_{2}, \cdots, u_{n}\right), p, S, e$ and $q$ represent fluid density, velocity, pressure, stress tensor, specific internal energy per unit mass and heat flux, respectively. The equations $(1.1)_{1},(1.1)_{2}$ and $(1.1)_{3}$ are the consequence of conservation of mass, momentum and energy, respectively.

To complete the system (1.1), we need to impose constitutive assumptions on $p$, $S, e$ and $q$. First, we assume the fluid to be a Newtonian fluid, that is,

$$
S=\mu\left(\nabla u+(\nabla u)^{T}\right)+\mu^{\prime} \nabla \operatorname{div} u,
$$

where $\mu$ and $\mu^{\prime}$ are the coefficient of viscosity and the second coefficient of viscosity, respectively, satisfying

$$
\mu>0, \mu^{\prime}+\frac{2}{n} \mu \geq 0
$$

The heat flux $q$ is assumed to satisfy

$$
\tau \partial_{t} q+q+\kappa \nabla \theta=0
$$


which represents Cattaneo's law (Maxwell's law, ...) and gives rise to heat waves with finite propagation speed. Here, $\tau>0$ is the constant relaxation time and $\kappa>0$ is the constant heat conductivity. Moreover, in this paper, we consider the general equations of state and assume that the pressure $p=p(\rho, \theta)$ and $e=e(\rho, \theta)$ are smooth functions of $(\rho, \theta)$ satisfying

$$
\rho^{2} e_{\rho}(\rho, \theta)=p(\rho, \theta)-\theta p_{\theta}(\rho, \theta),
$$

where $\theta$ denotes the absolute temperature. In particular, the case of a polytropic gas $p=R \rho \theta, e=c_{v} \theta$ is included here.

We consider the Cauchy problem for the functions

$$
(\rho, u, \theta, q): \mathbb{R}^{n} \times[0,+\infty) \rightarrow \mathbb{R}_{+} \times \mathbb{R}^{n} \times \mathbb{R}_{+} \times \mathbb{R}^{n}
$$

with initial condition

$$
(\rho(x, 0), u(x, 0), \theta(x, 0), q(x, 0))=\left(\rho_{0}, u_{0}, \theta_{0}, q_{0}\right) .
$$

For the limit case $\tau=0$, the system (1.1)-(1.3) is exactly the system of classical compressible Navier-Stokes equations, in which the relation between the heat flux and the temperature is governed by Fourier's law,

$$
q=-\kappa \nabla \theta \text {. }
$$

Because of its physical importance and mathematical challenging, the well-posed theory has been widely studied for the system (1.1), (1.2) combined with Fourier's law (1.6), see $[1,2,3,4,6,8,11,12,14,15,16,17,18,21,23]$. In particular, the local existence and uniqueness of smooth solutions was established by Serrin [21] and Nash [18] for initial data far away from vacuum. Later, Matsumura and Nishida [16] got global smooth solutions for small initial data without vacuum. For large data, Xin [23], Cho and Jin [1] showed that smooth solutions must blow up in finite time if the initial data has a vacuum state. The existence of global non-vacuum smooth solutions for large data is a famous open problem in fluid dynamics.

Although Fourier's law plays an important role in experimental and applied physics, it has the drawback of an inherent infinite propagation speed of signals. Cattaneo's law was among one of the physical laws describing the finite speed of heat conduction. It has been widely used in thermoelasticity which results in the second sound phenomenon, see $[7,19,20]$ and the references cited therein. However, a rigorous mathematical theory for the compressible Navier-Stokes system has not been established with heat conduction described by Catteneo's law. This is the aim of the presented paper. Note that it is not obvious that the results which hold for Fourier's law also hold for Cattaneo's law. Indeed and for example, Fernández Sare and Racke [5] showed that, for certain Timoshenko-type thermoelastic system, Fourier's law preserve the property of exponential stability while Cattaneo's law destroys such a property.

Moreover and naturally, it is interesting to study the relaxation limit $\tau \rightarrow 0$ for the system (1.1)-(1.5). As said above, for $\tau=0$ in (1.3), the system turns into 
the classical compressible Navier-Stokes equation. We will show this convergence rigorously and also obtain the convergence order with respect to $\tau$.

Now, we introduce some notation. Denote by $W^{m, p}(\Omega), 0 \leq m \leq \infty, 1 \leq p \leq \infty$, the usual Sobolev space with norm $\|\cdot\|_{W^{m, p}}$. For convenience, $H^{m}(\Omega)$ and $L^{p}(\Omega)$ stand for $W^{m, 2}(\Omega)$ and $W^{0, p}(\Omega)$ with norms $\|\cdot\|_{m}$ and $\|\cdot\|_{L^{p}}$, respectively. For $p=2$, we denote the norm $\|\cdot\|_{L^{2}}$ by $\|\cdot\|$. Denote by $G:=\mathbb{R}_{+} \times \mathbb{R}^{n} \times \mathbb{R}_{+} \times \mathbb{R}^{n}$ the physical state space.

The outline of this paper is as follows. We first show a local existence theorem by transforming the system (1.1)-(1.5) into a symmetric hyperbolic-parabolic type system. Then, for small $\tau$, we prove that the system satisfies the so-called Kawashima condition, and therefore we get a global solution for small initial data. Finally, we establish the convergence, as $\tau \rightarrow 0$, to the classical compressible Navier-Stokes system.

\section{LOCAL AND GLOBAL WELL-POSEDNESS}

In this part, we consider the local and global well-posedness for the system (1.1)(1.5). For this end, we need the following assumptions

- A.1. The initial data satisfy

$$
\left\{\left(\rho_{0}, u_{0}, \theta_{0}, q_{0}\right)(x): x \in \mathbb{R}^{n}\right\} \subset\left[\rho_{*}, \rho^{*}\right] \times\left[-C_{1}, C_{1}\right]^{n} \times\left[\theta_{*}, \theta^{*}\right] \times\left[-C_{1}, C_{1}\right]^{n}:=G_{0},
$$

where $C_{1}>0$ as well as $0<\rho_{*}<1<\rho^{*}<\infty$ and $0<\theta_{*}<1<\theta^{*}<\infty$ are constants.

- A.2. For each given $G_{1}$ satisfying $G_{0} \subset \subset G_{1} \subset \subset G, \forall(\rho, u, \theta, q) \in G_{1}$, the pressure $p$ and the internal energy $e$ satisfy

$$
p(\rho, \theta), p_{\theta}(\rho, \theta), p_{\rho}(\rho, \theta), e_{\theta}(\rho, \theta)>C\left(G_{1}\right)>0,
$$

where $C\left(G_{1}\right)$ is a positive constants depending on $G_{1}$.

For the standard assumption A.2 see for example [9, 17].

Theorem 2.1. (Local existence) Let $n \geq 1$ and $s \geq s_{0}+1$, with $s_{0} \geq\left[\frac{n}{2}\right]+1$, be integers. Suppose that the assumptions A.1 and A.2 hold and that the initial data $\left(\rho_{0}-1, u_{0}, \theta_{0}-1, q_{0}\right)$ are in $H^{s}$. Then, for each convex open subset $G_{1}$ satisfying $G_{0} \subset \subset G_{1} \subset \subset G$, there exists $T>0$ such that system (1.1)-(1.5) has unique classical solution $\left(\rho^{\tau}, u^{\tau}, \theta^{\tau}, q^{\tau}\right)$ satisfying

$$
\begin{array}{r}
\left(\rho^{\tau}-1, \theta^{\tau}-1, q^{\tau}\right) \in C\left([0, T], H^{s}\right) \cap C^{1}\left([0, T], H^{s-1}\right), \\
u^{\tau} \in C\left([0, T], H^{s}\right) \cap C^{1}\left([0, T], H^{s-2}\right)
\end{array}
$$

and

$$
\left(\rho^{\tau}, u^{\tau}, \theta^{\tau}, q^{\tau}\right)(x, t) \in G_{1}, \quad \forall(x, t) \in \mathbb{R}^{n} \times[0, T]
$$


Proof. We shall write the system (1.1) in symmetric form and use the classical theory of symmetric hyperbolic-parabolic system to prove Theorem 2.1.

By using equations $(1.1)_{1}$ and (1.2)-(1.4), the system (1.1) can be written as

$$
\left\{\begin{array}{l}
\partial_{t} \rho+u \nabla \rho+\rho \operatorname{div} u=0, \\
\rho \partial_{t} u+\rho u \nabla u+p_{\theta} \nabla \theta+p_{\rho} \nabla \rho=\mu \Delta u+\left(\mu+\mu^{\prime}\right) \nabla \operatorname{div} u, \\
\rho e_{\theta} \partial_{t} \theta+\rho e_{\theta} u \nabla \theta+\theta p_{\theta} \operatorname{div} u+\operatorname{div} q=\frac{\mu}{2}\left|\nabla u+(\nabla u)^{T}\right|^{2}+\mu^{\prime}|\operatorname{div} u|^{2}, \\
\tau \partial_{t} q+q+\kappa \nabla \theta=0,
\end{array}\right.
$$

with initial conditions

$$
(\rho(x, 0), u(x, 0), \theta(x, 0), q(x, 0))=\left(\rho_{0}, u_{0}, \theta_{0}, q_{0}\right) .
$$

Let $\omega:=(\rho, u, \theta, q)$. Then we have

$$
A^{0}(\omega) \partial_{t} \omega+\Sigma A^{j}(\omega) \partial_{x_{j}} \omega-\Sigma B^{j k}(\omega) \partial_{x_{j} x_{k}}^{2} \omega+L(\omega) \omega=g\left(\omega, D_{x} \omega\right),
$$

where $\Sigma \ldots$ always stands for $\sum_{j=1}^{n}$, and where

$$
\begin{aligned}
A^{0}(\omega)= & \left(\begin{array}{cccc}
\frac{c^{2}}{\rho} & 0 & 0 & 0 \\
0 & \rho I_{n} & 0 & 0 \\
0 & 0 & \frac{\rho e_{\theta}}{\theta} & 0 \\
0 & 0 & 0 & \frac{\tau}{\kappa \theta}
\end{array}\right), \Sigma A^{j} \xi_{j}=\left(\begin{array}{cccc}
\frac{c^{2}}{\rho} u \cdot \xi & c^{2} \xi & 0 & 0 \\
c^{2} \xi^{T} & \rho(u \cdot \xi) I_{n} & p_{\theta} \xi^{T} & 0 \\
0 & p_{\theta} \xi & \frac{\rho e_{\theta}}{\theta} u \cdot \xi & \frac{\xi}{\theta} \\
0 & 0 & \frac{\xi^{T}}{\theta} & 0
\end{array}\right), \\
\Sigma B^{j k} \xi_{j} \xi_{k} & =\left(\begin{array}{cccc}
0 & 0 & 0 & 0 \\
0 & \mu I_{n}+\left(\mu+\mu^{\prime}\right) \xi^{T} \xi & 0 & 0 \\
0 & 0 & 0 & 0 \\
0 & 0 & 0 & 0
\end{array}\right), L(\omega)=\left(\begin{array}{cccc}
0 & 0 & 0 & 0 \\
0 & 0 & 0 & 0 \\
0 & 0 & 0 & 0 \\
0 & 0 & 0 & \frac{1}{\kappa \theta} I_{n}
\end{array}\right) \\
g\left(\omega, D_{x} \omega\right) & =\left(\begin{array}{c}
0 \\
\frac{\mu}{2 \theta}\left|\nabla u+(\nabla u)^{T}\right|^{2}+\frac{\mu^{\prime}}{\theta}|\operatorname{div} u|^{2} \\
0
\end{array}\right), c^{2}=p_{\rho}, \xi=\left(\xi_{1}, \xi_{2}, \cdots, \xi_{n}\right) \in \mathcal{S}^{n-1} .
\end{aligned}
$$

By assumption A.2, one can see that for all $\omega \in G_{1}$ satisfying $G_{0} \subset \subset G_{1} \subset \subset G$, $A^{0}(\omega)$ is a positive symmetric matrix and $A^{j}(\omega)$ is a symmetric matrix for each $j$, while $\Sigma B^{j k}(\omega) \xi_{j} \xi_{k}$ is a semi-positive symmetric matrix. So, the local existence theorem follows from [13] (or see [10]) immediately.

Theorem 2.2. (Global existence) Let $n \geq 2$ and $s \geq s_{0}+1$, with $s_{0} \geq\left[\frac{n}{2}\right]+1$, be integers. Suppose that $0<\tau<\frac{2 \kappa}{p_{\theta}(1,1)^{2}}$ and $\left(\rho_{0}-1, u_{0}, \theta_{0}-1, q_{0}\right) \in H^{s}$. Then there exists a positive constants $\delta$ such that if $\left\|\left(\rho_{0}-1, u_{0}, \theta_{0}-1, q_{0}\right)\right\|_{s} \leq \delta$, then there exists a global unique solution $\left(\rho^{\tau}, u^{\tau}, \theta^{\tau}, q^{\tau}\right)$ of system (1.1)-(1.5) satisfying

$$
\begin{array}{r}
\left(\rho^{\tau}-1, \theta^{\tau}-1, q^{\tau}\right) \in C\left([0, \infty), H^{s}\right) \cap C^{1}\left([0, \infty), H^{s-1}\right), \\
u^{\tau} \in C\left([0, \infty), H^{s}\right) \cap C^{1}\left(\left[0, \infty, H^{s-2}\right) .\right.
\end{array}
$$


Proof. One can use the Kawashima condition to prove small global existence. Linearizing the above system around the steady state $\bar{\omega}=(\bar{\rho}, \bar{u}, \bar{\theta}, \bar{q}):=(1,0,1,0)$, one has

$$
A^{0}(\bar{\omega}) \partial_{t} \omega+\Sigma A^{j}(\bar{\omega}) \partial_{x_{j}} \omega-\Sigma B^{j k}(\bar{\omega}) \partial_{x_{j} x_{k}}^{2} \omega+L(\bar{\omega}) \omega=0
$$

where

$$
\begin{gathered}
A^{0}(\bar{\omega})=\left(\begin{array}{cccc}
\bar{c}^{2} & 0 & 0 & 0 \\
0 & I_{n} & 0 & 0 \\
0 & 0 & \bar{e}_{\theta} & 0 \\
0 & 0 & 0 & \frac{\tau}{\kappa}
\end{array}\right), \Sigma A^{j}(\bar{\omega}) \xi_{j}=\left(\begin{array}{cccc}
0 & \bar{c}^{2} \xi & 0 & 0 \\
\bar{c}^{2} \xi^{T} & 0 & \bar{p}_{\theta} \xi^{T} & 0 \\
0 & \bar{p}_{\theta} \xi & 0 & \xi \\
0 & 0 & \xi^{T} & 0
\end{array}\right), \\
\Sigma B^{j k}(\bar{\omega}) \xi_{j} \xi_{k}=\left(\begin{array}{cccc}
0 & 0 & 0 & 0 \\
0 & \mu I_{n}+\left(\mu+\mu^{\prime}\right) \xi^{T} \xi & 0 & 0 \\
0 & 0 & 0 & 0 \\
0 & 0 & 0
\end{array}\right), L(\bar{\omega})=\left(\begin{array}{cccc}
0 & 0 & 0 & 0 \\
0 & 0 & 0 & 0 \\
0 & 0 & 0 & 0 \\
0 & 0 & 0 & \frac{1}{\kappa} I_{n}
\end{array}\right), \\
\bar{c}=c(1,1), \bar{p}_{\theta}=p_{\theta}(1,1), \bar{e}_{\theta}=e_{\theta}(1,1), \xi=\left(\xi_{1}, \xi_{2}, \cdots, \xi_{n}\right) \in \mathcal{S}^{n-1} .
\end{gathered}
$$

We choose $K^{j}$ such that

$$
\Sigma K^{j} \xi_{j}=\alpha\left(\begin{array}{cccc}
0 & \bar{c}^{2} \xi & 0 & 0 \\
-\xi^{T} & 0 & 0 & 0 \\
0 & 0 & 0 & \frac{\kappa}{\tau} \xi \\
0 & 0 & -\frac{\xi^{T}}{e_{\theta}} & 0
\end{array}\right)
$$

where $\alpha>0$ will be chosen later. Then, simple calculations imply

$$
\Sigma K^{j} \xi_{j} A^{0}=\alpha\left(\begin{array}{cccc}
0 & \bar{c}^{2} \xi & 0 & 0 \\
-\bar{c}^{2} \xi^{T} & 0 & 0 & 0 \\
0 & 0 & 0 & \xi \\
0 & 0 & -\xi^{T} & 0
\end{array}\right)
$$

and

$$
\Sigma K^{j} A^{k} \xi_{j} \xi_{k}=\alpha\left(\begin{array}{cccc}
\bar{c}^{4} & 0 & \bar{p}_{\theta} \bar{c}^{2} & 0 \\
0 & -\bar{c}^{2} \xi^{T} \xi & 0 & 0 \\
0 & 0 & \frac{\kappa}{\tau} & 0 \\
0 & -\frac{\bar{p}_{\theta}}{\bar{e}_{\theta}} \xi^{T} \xi & 0 & -\frac{1}{\bar{e}_{\theta}} \xi^{T} \xi
\end{array}\right)
$$

In order to satisfy Kawashima's conditions from [13] (see also [22]) for proving the global existence for small data, we notice that there are no quadratic terms of the type $(\rho-1, u, \theta-1, q)^{2}$, but essentially only of type $|\nabla u|^{2}$. Hence one only has to 
check that

$$
\begin{aligned}
& \frac{1}{2} \Sigma\left\{K^{j} A^{k} \xi_{j} \xi_{k}+\left(K^{j} A^{k} \xi_{j} \xi_{k}\right)^{T}\right\}+\Sigma B^{j k} \xi_{j} \xi_{k}+L \\
& =\left(\begin{array}{cccc}
\alpha \bar{c}^{4} & 0 & \frac{1}{2} \alpha \bar{p}_{\theta} \bar{c}^{2} & 0 \\
0 & \mu I_{n}+\left(\left(\mu+\mu^{\prime}\right)-\alpha \bar{c}^{2}\right) \xi^{T} \xi & 0 & -\alpha \frac{\bar{p}_{\theta}}{2 \bar{e}_{\theta}} \xi^{T} \xi \\
\frac{1}{2} \alpha \bar{p}_{\theta} \bar{c}^{2} & 0 & \alpha \frac{\kappa}{\tau} & 0 \\
0 & -\alpha \frac{\bar{p}_{\theta}}{2 \bar{e}_{\theta}} \xi^{T} \xi & 0 & \frac{1}{\kappa} I_{n}-\alpha \frac{1}{\bar{e}_{\theta}} \xi^{T} \xi
\end{array}\right)=: M
\end{aligned}
$$

is a positive definite matrix for any $\xi=\left(\xi_{1}, \xi_{2}, \cdots, \xi_{n}\right) \in \mathcal{S}^{n-1}$. In fact, let $\eta=$ $\left(\eta_{1}, \eta_{2}, \eta_{3}, \eta_{4}\right) \in \mathbb{R}^{8}$ where $\eta_{1}, \eta_{3} \in \mathbb{R}^{1}$ and $\eta_{2}, \eta_{4} \in \mathbb{R}^{3}$. Then we have

$$
\begin{aligned}
& \eta M \eta^{T}=\left(\eta_{1} \alpha \bar{c}^{4}+\frac{1}{2} \eta_{3} \alpha \bar{p}_{\theta} \bar{c}^{2}\right) \eta_{1}+ \\
& \left(\eta_{2} N+\eta_{4}\left(-\alpha \frac{\bar{p}_{\theta}}{2 \bar{e}_{\theta}} \xi^{T} \xi\right)\right) \eta_{2}+\left(\frac{1}{2} \eta_{1} \alpha \bar{p}_{\theta} \bar{c}^{2}+\eta_{3} \alpha \frac{\kappa}{\tau}\right) \eta_{3}+\left(-\eta_{2} \alpha \frac{\bar{p}_{\theta}}{2 \bar{e}_{\theta}} \xi^{T} \xi+\eta_{4} Q\right) \eta_{4},
\end{aligned}
$$

where $N=\mu I_{n}+\left(\left(\mu+\mu^{\prime}\right)-\alpha \bar{c}^{2}\right) \xi^{T} \xi$ and $Q=\frac{1}{\kappa} I_{n}-\alpha \frac{1}{\bar{e}_{\theta}} \xi^{T} \xi$. If $0<\tau<\frac{2 \kappa}{\bar{p}_{\theta}^{2}}$, we know that

$$
\triangle:=\bar{p}_{\theta}^{2}-4 \frac{\kappa}{\tau}<0
$$

So, for any $\alpha>0$, we have

$$
\alpha \bar{c}^{4} \eta_{1}^{2}+\alpha \bar{c}^{2} \bar{p}_{\theta} \eta_{1} \eta_{3}+\alpha \frac{\kappa}{\tau} \eta_{3}^{2}>0 .
$$

On the other hand, for small $\alpha$, we know that the matrix $N$ and $Q$ are positive definite. So, we can choose $\alpha$ small enough such that

$$
\eta_{2} N \eta_{2}-\alpha \eta_{4} \frac{\bar{p}_{\theta}}{2 \bar{e}_{\theta}} \xi^{T} \xi \eta_{2}+\eta_{4} Q \eta_{4}>0
$$

Therefore, for sufficiently small $\alpha$, we derive that $\eta M \eta^{T}>0$ for any $\eta \in \mathbb{R}^{8}$ which implies that $M$ is positive definite. This completes the proof.

\section{Relaxation limit: COnVERgence For $\tau \rightarrow 0$}

In this part, we show the uniform convergence of the relaxed system $(\tau>0)$ to the classical compressible Navier-Stokes equations $(\tau=0)$. To this end, we need the natural compatibility condition on the initial data, that is,

$$
q_{0}=-\kappa \nabla \theta_{0}
$$

which be assumed in this section. Let $G_{1}$ be given satisfying $G_{0} \subset \subset G_{1} \subset \subset G$. Define $T_{\tau}=\sup \left\{T>0 ;\left(\rho^{\tau}-1, v^{\tau}, \theta^{\tau}-1, q^{\tau}\right) \in C\left([0, T], H^{s}\right), \quad\left(\rho^{\tau}, v^{\tau}, \theta^{\tau}, q^{\tau}\right)(x, t) \in\right.$ $\left.G_{1}, \quad \forall(x, t) \in \mathbb{R}^{n} \times[0, T]\right\}$. Then we have the following theorem.

Theorem 3.1. Let $(\rho, u, \theta)$ be a smooth solution to the classical compressible NavierStokes equations with $(\rho(x, 0), u(x, 0), \theta(x, 0))=\left(\rho_{0}, u_{0}, \theta_{0}\right)$ satisfying $\rho \in C\left(\left[0, T_{*}\right], H^{s+3}\right) \cap C^{1}\left(\left[0, T_{*}\right], H^{s+2}\right),(u, \theta) \in C\left(\left[0, T_{*}\right], H^{s+3}\right) \cap C^{1}\left(\left[0, T_{*}\right], H^{s+1}\right)$ 
with $T_{*}>0$ finite. Then there are positive constants $\tau_{0}$ and $C$ such that for $\tau \leq \tau_{0}$,

$$
\left\|\left(\rho^{\tau}, u^{\tau}, \theta^{\tau}\right)(t, \cdot)-(\rho, u, \theta)(t, \cdot)\right\|_{s} \leq C \tau
$$

and

$$
\left\|\left(q^{\tau}+\kappa \nabla \theta\right)(t, \cdot)\right\|_{s} \leq C \tau^{\frac{1}{2}}
$$

for $t \in\left[0, \min \left\{T_{*}, T_{\tau}\right\}\right)$, where $C$ does not depend on $\tau$.

Theorem 3.1 in particular implies that $T_{\tau}$ is independent of $\tau$.

Theorem 3.2. Under the condition of Theorem 3.1, for any $G_{1}$ satisfying

$$
G_{0} \cup \tilde{G} \subset \subset G_{1} \subset \subset G
$$

where $\tilde{G}=\left\{\cup(\rho, u, \theta,-\kappa \nabla \theta)(x, t),(x, t) \in \mathbb{R}^{n} \times\left[0, T_{*}\right]\right\}$, we have that $T_{\tau}>T_{*}$ holds for $\tau>0$ sufficiently small.

Proof. The proof of Theorem 3.2 follows the arguments in [24]. For the reader's convenience we sketch it here.

Suppose, there is a $G_{1}$ satisfying (3.3) and a sequence $\left\{\tau_{k}\right\}_{k \geq 1}$ such that $\lim _{k \rightarrow \infty} \tau_{k}=0$ and $T_{\tau_{k}}=T_{\tau_{k}}\left(G_{1}\right) \leq T_{*}$. Then there exists $\tilde{G}$ satisfying

$$
\cup_{x, t}\left\{(\rho, u, \theta,-\kappa \nabla \theta),(x, t) \in \mathbb{R}^{n} \times\left[0, T_{*}\right]\right\} \subset \subset \tilde{G} \subset \subset G_{1} .
$$

Moreover, from Sobolev's imbedding theorem and Theorem 3.1, we deduce that

$$
\begin{aligned}
& \left|\left(\rho^{\tau}, u^{\tau}, \theta^{\tau}, q^{\tau}\right)-(\rho, u, \theta,-\kappa \nabla \theta)\right| \\
& \leq C\left\|\left(\rho^{\tau}, u^{\tau}, \theta^{\tau}, q^{\tau}\right)-(\rho, u, \theta,-\kappa \nabla \theta)\right\|_{s} \leq C \tau^{\frac{1}{2}} .
\end{aligned}
$$

Thus, there exists a $k$ such that $\left(\rho^{\tau}, u^{\tau}, \theta^{\tau}, q^{\tau}\right) \in \tilde{G}$ for all $(x, t) \in \mathbb{R}^{n} \times\left[0, T_{\tau_{k}}\right)$. On the other hand, it follows from

$$
\begin{aligned}
& \left\|\left(\rho^{\tau}-1, u^{\tau}, \theta^{\tau}-1, q^{\tau}\right)\right\|_{s} \\
& \leq\left\|\left(\rho^{\tau}, u^{\tau}, \theta^{\tau}, q^{\tau}\right)-(\rho, u, \theta,-\kappa \nabla \theta)\right\|_{s}+\|(\rho-1, u, \theta-1,-\kappa \nabla \theta)\|_{s} \\
& \leq C \tau_{k}^{\frac{1}{2}}+\|(\rho-1, u, \theta-1,-\kappa \nabla \theta)\|_{s}
\end{aligned}
$$

that $\left\|\left(\rho^{\tau}-1, u^{\tau}, \theta^{\tau}-1, q^{\tau}\right)\right\|_{s}$ is uniformly bounded with respect to $t \in\left[0, T_{\tau_{k}}\right)$. Now, we could apply Theorem 2.1 at a time $t$ less than $T_{\tau_{k}}$ ( $k$ is fixed here) to continue the solution beyond $T_{\tau_{k}}\left(G_{1}\right)$ which is a contradiction and the proof is completed.

Remark 3.1. We note that if the initial data are sufficiently small, there exists a global solution for classical Compressible Navier-Stokes equations, see [16]. Therefore, we can establish a convergence results for any fixed $T>0$ for small data.

Remark 3.2. A global convergence theorem on $[0, \infty)$ can not be obtained from the above results. One would have to spend considerably more efforts to get this result (if at all). 
Proof of Theorem 3.1:

We introduce the variable $q:=-\kappa \nabla \theta$ and define

$$
\rho^{d}:=\frac{\rho^{\tau}-\rho}{\tau}, u^{d}=\frac{u^{\tau}-u}{\tau}, \theta^{d}=\frac{\theta^{\tau}-\theta}{\tau}, q^{d}=\frac{q^{\tau}-q}{\tau} .
$$

We want to show that, for small $\tau$ and for $t<\min \left\{T_{*}, T_{\tau}\right\}$,

$$
\left\|\left(\rho^{d}, u^{d}, \theta^{d}\right)(t, \cdot)\right\|_{s} \leq C, \quad\left\|\sqrt{\tau} q^{d}(t, \cdot)\right\|_{s} \leq C,
$$

where $C>0$ denotes constants not depending on $\tau$ or $t$. The equations for the difference $\left(\rho^{d}, u^{d}, \theta^{d}, q^{d}\right)$ can be written as

$$
\begin{aligned}
& \partial_{t} \rho^{d}+u^{\tau} \nabla \rho^{d}+u^{d} \nabla \rho+\rho^{\tau} \operatorname{div} u^{d}+\rho^{d} \operatorname{div} u=0 \\
& \rho^{\tau} \partial_{t} u^{d}+\rho^{d} \partial_{t} u+\rho^{\tau} u^{\tau} \nabla u^{d}+p_{\theta}^{\tau} \nabla \theta^{d}+p_{\rho}^{\tau} \nabla \rho^{d} \\
& +\frac{1}{\tau}\left\{\left(\rho^{\tau} u^{\tau}-\rho u\right) \nabla u+\left(p_{\rho}^{\tau}-p_{\rho}\right) \nabla \rho+\left(p_{\theta}^{\tau}-p_{\theta}\right) \nabla \theta\right\}=\mu \Delta u^{d}+\left(\mu+\mu^{\prime}\right) \nabla \operatorname{div} u^{d} \\
& \rho^{\tau} e_{\theta}^{\tau} \partial_{t} \theta^{d}+\rho^{\tau} e_{\theta}^{\tau} u^{\tau} \nabla \theta^{d}+\theta^{\tau} p_{\theta}^{\tau} \operatorname{div} u^{d}+\operatorname{div} q^{d} \\
& \quad-\mu\left(\nabla u^{\tau}+\left(\nabla u^{\tau}\right)^{T}+\nabla u+(\nabla u)^{T}\right) \nabla u^{d}-\mu^{\prime}\left(\operatorname{div} u^{\tau}+\operatorname{div} u\right) \operatorname{div} u^{d} \\
& =\frac{1}{\tau}\left\{\left(\rho^{\tau} e_{\theta}^{\tau}-\rho e_{\theta}\right) \partial_{t} \theta+\left(\rho^{\tau} e_{\theta}^{\tau} u^{\tau}-\rho e_{\theta} u\right) \nabla \theta+\left(\theta^{\tau} p_{\theta}^{\tau}-\theta p_{\theta}\right) \operatorname{div} u\right\} \\
& \tau \partial_{t} q^{d}+q^{d}+\kappa \nabla \theta^{d}=-\partial_{t} q .
\end{aligned}
$$

In order to use energy methods, we rewrite the above system as

$$
\begin{aligned}
& \partial_{t} \rho^{d}+u^{\tau} \nabla \rho^{d}+\rho^{\tau} \operatorname{div} u^{d}=-u^{d} \nabla \rho-\rho^{d} \operatorname{div} u=: f_{1}, \\
& \partial_{t} u^{d}+u^{\tau} \nabla u^{d}+\frac{p_{\theta}^{\tau}}{\rho^{\tau}} \nabla \theta^{d}+\frac{p_{\rho}^{\tau}}{\rho^{\tau}} \nabla \rho^{d}-\frac{1}{\rho^{\tau}}\left(\mu \triangle u^{d}+\left(\mu+\mu^{\prime}\right) \nabla \operatorname{div} u^{d}\right) \\
& =-\frac{1}{\rho^{\tau}} \rho^{d} u_{t}-\frac{1}{\tau \rho^{\tau}}\left\{\left(\rho^{\tau} u^{\tau}-\rho u\right) \nabla u+\left(p_{\rho}^{\tau}-p_{\rho}\right) \nabla \rho+\left(p_{\theta}^{\tau}-p_{\theta}\right) \nabla \theta\right\}=: f_{2}, \\
& \partial_{t} \theta^{d}+u^{\tau} \nabla \theta^{d}+\frac{\theta^{\tau} p_{\theta}^{\tau}}{\rho^{\tau} e_{\theta}^{\tau}} \operatorname{div} u^{d}+\frac{1}{\rho^{\tau} e_{\theta}^{\tau}} \operatorname{div} q^{d} \\
& =\frac{1}{\tau \rho^{\tau} e_{\theta}^{\tau}}\left\{\left(\rho^{\tau} e_{\theta}^{\tau}-\rho e_{\theta}\right) \partial_{t} \theta+\left(\rho^{\tau} e_{\theta}^{\tau} u^{\tau}-\rho e_{\theta} u\right) \nabla \theta+\left(\theta^{\tau} p_{\theta}^{\tau}-\theta p_{\theta}\right) \operatorname{div} u\right\} \\
& +\frac{\mu}{\rho^{\tau} e_{\theta}^{\tau}}\left(\nabla u^{\tau}+\left(\nabla u^{\tau}\right)^{T}+\nabla u+(\nabla u)^{T}\right) \nabla u^{d}+\frac{\mu^{\prime}}{\rho^{\tau} e_{\theta}^{\tau}}\left(\operatorname{div} u^{\tau}+\operatorname{div} u\right) \operatorname{div} u^{d}=: f_{3}, \\
& \tau \partial_{t} q^{d}+q^{d}+\kappa \nabla \theta^{d}=-\partial_{t} q=: f_{4} .
\end{aligned}
$$

Define

$$
E:=\sup _{0 \leq t \leq T}\|(\rho, u, \theta)\|_{H^{s+3}}+\sup _{0 \leq t \leq T}\left\|\rho_{t}\right\|_{H^{s+2}}+\sup _{0 \leq t \leq T}\left\|\left(u_{t}, \theta_{t}\right)\right\|_{H^{s+1}}
$$


and

$$
E^{d}:=\sup _{0 \leq t \leq T}\left\|\left(\rho^{d}, u^{d}, \theta^{d}, \sqrt{\tau} q^{d}\right)\right\|_{H^{s}}
$$

Note that

$$
E \leq C
$$

and

$$
\left\|\left(\rho^{\tau}, u^{\tau}, \theta^{\tau}\right)\right\|_{s} \leq C+\tau E^{d},\left\|q^{\tau}\right\|_{s} \leq C+\sqrt{\tau} E^{d} .
$$

We need the following two lemmas to prove our theorem.

Lemma 3.3. For $0 \leq|\alpha| \leq s$, we have the following estimates

$$
\begin{array}{r}
\left\|\nabla^{\alpha} f_{1}\right\| \leq C E^{d}, \\
\left\|\nabla^{\alpha} f_{2}\right\| \leq C\left(E^{d}+\tau\left(E^{d}\right)^{2}\right), \\
\left\|\nabla^{\alpha} f_{3}\right\| \leq C\left(E^{d}+\tau\left(E^{d}\right)^{2}+\tau^{2}\left(E^{d}\right)^{3}+\left\|\nabla^{\alpha+1} u^{d}\right\|+\tau E^{d}\left\|\nabla^{\alpha+1} u^{d}\right\|\right) .
\end{array}
$$

Proof. By Sobolev's imbedding theorem and Moser inequalities, using (3.17), we have

$$
\begin{aligned}
& \left\|\nabla^{\alpha} f_{1}\right\|=\| \nabla^{\alpha}\left(-u^{d} \nabla \rho-\rho^{d} \operatorname{div} u \|\right. \\
& \leq\|\nabla \rho\|_{L^{\infty}}\left\|\nabla^{\alpha} u^{d}\right\|+\left\|u^{d}\right\|_{L^{\infty}}\left\|\nabla^{\alpha+1} \rho\right\|+\|\operatorname{div} u\|_{L^{\infty}}\left\|\nabla^{\alpha} \rho^{d}\right\|+\left\|\rho^{d}\right\|_{L^{\infty}}\left\|\nabla^{\alpha+1} u\right\| \\
& \leq C E^{d} .
\end{aligned}
$$

Therefore, (3.19) holds.

Remember that both $(\rho, u, \theta)$ and $\left(\rho^{\tau}, u^{\tau}, \theta^{\tau}, q^{\tau}\right)$ take values in a convex compact subset of the state space, we have

$$
\begin{aligned}
& \left\|\nabla^{\alpha}\left(-\frac{1}{\rho^{\tau}} \rho^{d} u_{t}\right)\right\| \\
& \leq\left\|u_{t}\right\|_{L^{\infty}}\left\|\nabla^{\alpha}\left(\frac{\rho^{d}}{\rho^{\tau}}\right)\right\|+\left\|\frac{\rho^{d}}{\rho^{\tau}}\right\|_{L^{\infty}}\left\|\nabla^{\alpha} u_{t}\right\| \\
& \leq C E^{d}+C\left\|\rho^{d}\right\|_{L^{\infty}}\left\|\nabla^{\alpha} \rho^{\tau}\right\| \leq C\left(E^{d}+\tau\left(E^{d}\right)^{2}\right) .
\end{aligned}
$$

Similarly, we have

$$
\left\|\nabla^{\alpha}\left(\frac{1}{\tau \rho^{\tau}}\left(\rho^{\tau} u^{\tau}-\rho u\right) \nabla u\right)\right\| \leq C\left(E^{d}+\tau\left(E^{d}\right)^{2}\right) .
$$

Recalling that $p(\rho, \theta)$ is a smooth function of $(\rho, \theta)$ and using the mean value theorem, we obtain

$$
\begin{aligned}
& \left\|\nabla^{\alpha}\left(\frac{1}{\tau \rho^{\tau}}\left(\left(p_{\rho}^{\tau}-p_{\rho}\right) \nabla \rho+\left(p_{\theta}^{\tau}-p_{\theta}\right) \nabla \theta\right)\right)\right\| \\
& \leq C\left\|\nabla^{\alpha}\left(\frac{1}{\rho^{\tau}}\left(\rho^{d}+\theta^{d}\right)\left(\nabla \rho+\nabla^{\theta}\right)\right)\right\| \leq C\left(E^{d}+\tau\left(E^{d}\right)^{2}\right) .
\end{aligned}
$$


Thus, (3.20) holds.

By assumption A.2 and using the mean value theorem, we have

$$
\begin{aligned}
& \left\|\nabla^{\alpha}\left(\frac{1}{\rho^{\tau} e_{\theta}^{\tau}}\left(\rho^{\tau} e_{\theta}^{\tau}-\rho e_{\theta}\right) \theta_{t}\right)\right\| \\
& \leq\left\|\nabla^{\alpha}\left(\frac{1}{\tau e_{\theta}^{\tau}}\left(e_{\theta}^{\tau}-e_{\theta}\right)\right)\right\|+\left\|\nabla^{\alpha}\left(\frac{\rho^{d}}{\rho^{\tau} e_{\theta}^{\tau}} e_{\theta} \theta_{t}\right)\right\| \\
& \leq C\left(E^{d}+\tau\left(E^{d}\right)^{2}\right),
\end{aligned}
$$

where we used the fact that

$$
\left\|\nabla^{\alpha}\left(\rho^{\tau} e_{\theta}^{\tau}\right)\right\| \leq\left\|\rho^{\tau}\right\|_{L^{\infty}}\left\|\nabla^{\alpha} e_{\theta}^{\tau}\right\|+\left\|e_{\theta}^{\tau}\right\|_{L^{\infty}}\left\|\nabla \rho^{\tau}\right\| \leq C+\tau E^{d} .
$$

Similarly, we get

$$
\left\|\nabla^{\alpha}\left(\frac{1}{\tau \rho^{\tau} e_{\theta}^{\tau}}\left(\theta^{\tau} p_{\theta}^{\tau}-\theta p_{\theta}\right) \operatorname{div} u\right)\right\| \leq C\left(E^{d}+\tau\left(E^{d}\right)^{2}\right)
$$

and

$$
\left\|\nabla^{\alpha}\left(\frac{1}{\tau \rho^{\tau} e_{\theta}^{\tau}}\left(\rho^{\tau} e_{\theta}^{\tau} u^{\tau}-\rho e_{\theta} u\right) \nabla \theta\right)\right\| \leq C\left(E^{d}+\tau\left(E^{d}\right)^{2}\right) .
$$

Moreover, we have

$$
\begin{aligned}
& \left\|\nabla^{\alpha}\left(\frac{\mu}{\rho^{\tau} e_{\theta}^{\tau}}\left(\nabla u^{\tau}+\left(\nabla u^{\tau}\right)^{T}+\nabla u+\nabla u^{T}\right) \nabla u^{d}\right)\right\| \\
& \leq C\left\|\nabla^{\alpha}\left(\frac{\mu}{\rho^{\tau} e_{\theta}^{\tau}}\left(\left(\nabla u+\tau \nabla u^{d}\right) \nabla u^{d}\right)\right)\right\| \\
& \leq\|\nabla u\|_{L^{\infty}}\left\|\nabla^{\alpha}\left(\frac{\mu}{\rho^{\tau} e_{\theta}^{\tau}} \nabla u^{d}\right)\right\|+\left\|\frac{\mu}{\rho^{\tau} e_{\theta}^{\tau}} \nabla u^{d}\right\|_{L^{\infty}}\|\nabla u\| \\
& +\tau\left\|\frac{\mu}{\rho^{\tau} e_{\theta}^{\tau}}\right\|_{L^{\infty}}\left\|\nabla^{\alpha}\left(\nabla u^{d}: \nabla u^{d}\right)\right\|+\tau\left\|\nabla u^{d}: \nabla u^{d}\right\|_{L^{\infty}}\left\|\nabla^{\alpha}\left(\frac{\mu}{\rho^{\tau} e_{\theta}^{\tau}}\right)\right\| \\
& \leq C\left(E^{d}+\tau\left(E^{d}\right)^{2}+\tau^{2}\left(E^{d}\right)^{3}+\left\|\nabla^{\alpha+1} u^{d}\right\|+\tau E^{d}\left\|\nabla^{\alpha+1} u^{d}\right\|\right) .
\end{aligned}
$$

Similarly, we get

$$
\begin{aligned}
& \left\|\nabla^{\alpha}\left(\frac{\mu^{\prime}}{\rho^{\tau} e_{\theta}^{\tau}}\left(\operatorname{div} u^{\tau}+\operatorname{div} u\right) \operatorname{div} u^{d}\right)\right\| \\
& \leq C\left(E^{d}+\tau\left(E^{d}\right)^{2}+\tau^{2}\left(E^{d}\right)^{3}+\left\|\nabla^{\alpha+1} u^{d}\right\|+\tau E^{d}\left\|\nabla^{\alpha+1} u^{d}\right\|\right) .
\end{aligned}
$$

So, (3.21) holds and this completes the proof.

Lemma 3.4. we have

$$
\frac{\mathrm{d}}{\mathrm{d} t}\left(E^{d}\right)^{2} \leq C\left(1+\left(E^{d}\right)^{2}+\tau\left(E^{d}\right)^{3}+\tau^{2}\left(E^{d}\right)^{4}\right)
$$


Proof. Applying $\nabla^{\alpha}$ to the equations (3.11)-(3.14) and multiplying the result by $\frac{p_{\rho}^{\tau}}{\rho^{\tau}} \nabla^{\alpha} \rho^{d}, \rho^{\tau} \nabla^{\alpha} u^{d}, \frac{\rho^{\tau} e_{\theta}^{\tau}}{\theta^{\tau}} \nabla^{\alpha} \theta^{d}, \frac{1}{\kappa \theta^{\tau}} \nabla^{\alpha} q^{d}$, respectively, we get

$$
\begin{aligned}
& \frac{1}{2} \frac{\mathrm{d}}{\mathrm{d} t} \int\left\{\frac{p_{\rho}^{\tau}}{\rho^{\tau}}\left(\nabla^{\alpha} \rho^{d}\right)^{2}+\rho^{\tau}\left(\nabla^{\alpha} u^{d}\right)^{2}+\frac{\rho^{\tau} e_{\theta}^{\tau}}{\theta^{\tau}}\left(\nabla^{\alpha} \theta^{d}\right)^{2}+\frac{\tau}{\kappa \theta^{\tau}}\left(\nabla^{\alpha} q^{d}\right)^{2}\right\} \mathrm{d} x \\
& +\int\left\{\mu\left(\nabla^{\alpha+1} u^{d}\right)^{2}+\left(\mu+\mu^{\prime}\right)\left(\nabla^{\alpha} \operatorname{div} u^{d}\right)^{2}+\frac{1}{\kappa \theta^{\tau}}\left(\nabla^{\alpha} q^{d}\right)^{2}\right\} \mathrm{d} x \\
& \leq \sum_{i=1}^{4}\left(F_{i}+T_{i}\right)+\sum_{i=1}^{9} G_{i}+D
\end{aligned}
$$

where

$$
\begin{aligned}
& F_{1}=\int \nabla^{\alpha} f_{1} \frac{p_{\rho}^{\tau}}{\rho^{\tau}} \nabla^{\alpha} \rho^{d} \mathrm{~d} x, F_{2}=\int \nabla^{\alpha} f_{2} \rho^{\tau} \nabla^{\alpha} u^{d} \mathrm{~d} x \\
& F_{3}=\int \nabla^{\alpha} f_{3} \frac{\rho^{\tau} e_{\theta}^{\tau}}{\theta^{\tau}} \nabla^{\alpha} \theta^{d} \mathrm{~d} x, F_{4}=\int \nabla^{\alpha} f_{4} \frac{\nabla^{\alpha} q^{d}}{\kappa \theta^{\tau}} \mathrm{d} x \\
& T_{1}=\int\left(\frac{p_{\rho}^{\tau}}{\rho^{\tau}}\right)_{t}\left(\nabla^{\alpha} \rho^{d}\right)^{2} \mathrm{~d} x, T_{2}=\int \rho_{t}^{\tau}\left(\nabla^{\alpha} u^{d}\right)^{2} \mathrm{~d} x, \\
& T_{3}=\int\left(\frac{\rho^{\tau} e_{\theta}^{\tau}}{\theta^{\tau}}\right)_{t}\left(\nabla^{\alpha} \theta^{d}\right)^{2} \mathrm{~d} x, T_{4}=\int\left(\frac{1}{\kappa \theta^{\tau}}\right)_{t}\left(\nabla^{\alpha} q^{d}\right)^{2} \mathrm{~d} x \\
& D=\int\left\{\nabla^{\alpha}\left(\frac{1}{\rho^{\tau}}\left(\mu \triangle u^{d}+\left(\mu+\mu^{\prime}\right) \nabla \operatorname{div} u^{d}\right)\right)-\frac{1}{\rho^{\tau}} \nabla^{\alpha}\left(\mu \triangle u^{d}+\left(\mu+\mu^{\prime}\right) \nabla \operatorname{div} u^{d}\right)\right\} \mathrm{d} x, \\
& G_{1}=\int \nabla^{\alpha}\left(u^{\tau} \nabla \rho^{d}\right) \frac{p_{\rho}^{\tau}}{\rho^{\tau}} \nabla^{\alpha} \rho^{d} \mathrm{~d} x, G_{2}=\int \nabla^{\alpha}\left(\rho^{\tau} \operatorname{div} u^{d}\right) \frac{p_{\rho}^{\tau}}{\rho^{\tau}} \nabla^{\alpha} \rho^{d} \mathrm{~d} x, \\
& G_{3}=\int \nabla^{\alpha}\left(u^{\tau} \nabla u^{d}\right) \rho^{\tau} \nabla^{\alpha} u^{d} \mathrm{~d} x, G_{4}=\int \nabla^{\alpha}\left(\frac{p_{\rho}^{\tau}}{\rho^{\tau}} \nabla \rho^{d}\right) \rho^{\tau} \nabla^{\alpha} u^{d} \mathrm{~d} x \\
& G_{5}=\int \nabla^{\alpha}\left(\frac{p_{\theta}^{\tau}}{\rho^{\tau}} \nabla \theta^{d}\right) \rho^{\tau} \nabla^{\alpha} u^{d} \mathrm{~d} x, G_{6}=\int \nabla^{\alpha}\left(u^{\tau} \nabla \theta^{d}\right) \frac{\rho^{\tau} e_{\theta}^{\tau}}{\theta^{\tau}} \nabla^{\alpha} \theta^{d} \mathrm{~d} x, \\
& G_{7}=\int \nabla^{\alpha}\left(\frac{\theta^{\tau} p_{\theta}^{\tau}}{\rho^{\tau} e_{\theta}^{\tau}} \operatorname{div} u^{d}\right) \frac{\rho^{\tau} e_{\theta}^{\tau}}{\theta^{\tau}} \nabla^{\alpha} \theta^{d} \mathrm{~d} x \\
& G_{8}=\int \nabla^{\alpha}\left(\frac{1}{\rho^{\tau} e_{\theta}^{\tau}} \operatorname{div} q^{d}\right) \frac{\rho^{\tau} e_{\theta}^{\tau}}{\theta^{\tau}} \nabla^{\alpha} \theta^{d} \mathrm{~d} x, G_{9}=\int \nabla^{\alpha}\left(\kappa \nabla \theta^{d}\right) \frac{\nabla^{\alpha} q^{d}}{\kappa \theta^{\tau}} \mathrm{d} x .
\end{aligned}
$$

From Lemma 3.3, we know that

$$
F_{i} \leq C\left(\left(E^{d}\right)^{2}+\tau\left(E^{d}\right)^{3}+\tau^{2}\left(E^{d}\right)^{4}\right)+\varepsilon\left\|\nabla^{\alpha+1} u^{d}\right\|^{2}
$$

$C$ depending on $\varepsilon$, for each $i=1,2,3$ and $F_{4} \leq C+\varepsilon\left\|\nabla^{\alpha} q^{d}\right\|^{2}$ (here we use that $q_{t}=-\kappa \nabla \theta_{t}$ and $\left.\left\|\theta_{t}\right\|_{s+1} \leq C\right)$. Now, we estimate $G_{i}$ for each $i$. 


$$
\begin{aligned}
& G_{1}=\int\left\{\left(\nabla^{\alpha}\left(u^{\tau} \nabla \rho^{d}\right)-u^{\tau} \nabla^{\alpha+1} \rho^{d}\right) \frac{p_{\rho}^{\tau}}{\rho^{\tau}} \nabla^{\alpha} \rho^{d}+\frac{u^{\tau} p_{\rho}^{\tau}}{\rho^{\tau}} \nabla^{\alpha+1} \rho^{d} \nabla^{\alpha} \rho^{d}\right\} \mathrm{d} x \\
& \leq C\left(\left\|\nabla \rho^{d}\right\|_{L^{\infty}}\left\|\nabla^{\alpha} u^{\tau}\right\|+\left\|\nabla u^{\tau}\right\|_{L^{\infty}}\left\|\nabla^{\alpha} \rho^{d}\right\|\right)\left\|\nabla^{\alpha} \rho^{d}\right\|_{s}+\left\|\nabla\left(\frac{u^{\tau} p_{\rho}^{\tau}}{\rho^{\tau}}\right)\right\|_{L^{\infty}}\left\|\nabla^{\alpha} \rho^{d}\right\|^{2} \\
& \leq C\left(\left(E^{d}\right)^{2}+\tau\left(E^{d}\right)^{3}\right) .
\end{aligned}
$$

$G_{6}$ can be estimated the same way.

$$
\begin{aligned}
& G_{2}+G_{4} \\
& =\int\left(\nabla^{\alpha}\left(\rho^{\tau} \operatorname{div} u^{d}\right)-\rho^{\tau} \operatorname{div} \nabla^{\alpha} u^{d}\right) \frac{p_{\rho}^{\tau}}{\rho^{\tau}} \nabla^{\alpha} \rho^{d} \mathrm{~d} x \\
& \quad+\int\left\{\nabla^{\alpha}\left(\frac{p_{\rho}^{\tau}}{\rho^{\tau}} \nabla \rho^{d}\right) \rho^{\tau} \nabla^{\alpha} u^{d}-p_{\rho}^{\tau} \nabla^{\alpha+1} \rho^{d} \nabla^{\alpha} u^{d}\right\} \mathrm{d} x \\
& \leq C\left\|\left(\operatorname{div} u^{d}, \nabla \rho^{d}\right)\right\|_{L^{\infty}}\left(\left\|\nabla^{\alpha} \rho^{\tau}\right\|+\left\|\nabla\left(\frac{p_{\rho}^{\tau}}{\rho^{\tau}}\right)\right\|\right)\left(\left\|\nabla^{\alpha} \rho^{d}\right\|+\left\|\nabla^{\alpha} u^{d}\right\|\right) \\
& +\left\|\left(\nabla \rho^{d}, \nabla p_{\rho}^{\tau}, \frac{p_{\rho}^{\tau}}{\rho^{\tau}}\right)\right\|_{L^{\infty}}\left\|\nabla^{\alpha} u^{d}\right\|\left\|\nabla^{\alpha} \rho^{d}\right\| \leq C\left(\left(E^{d}\right)^{2}+\tau\left(E^{d}\right)^{3}\right) .
\end{aligned}
$$

$G_{5}+G_{7}$ and can also be estimated similarly, while

$$
\begin{gathered}
G_{8}+G_{9} \\
=\int\left\{\nabla^{\alpha}\left(\frac{1}{\rho^{\tau} e_{\theta}^{\tau}} \operatorname{div} q^{d}\right) \frac{\rho^{\tau} e_{\theta}^{\tau}}{\theta^{\tau}} \nabla^{\alpha} \theta^{d}-\frac{1}{\theta^{\tau}} \nabla^{\alpha} \operatorname{div} q^{d} \nabla^{\alpha} \theta^{d}-\nabla\left(\frac{1}{\theta^{\tau}}\right) \nabla^{\alpha} \theta^{d} \nabla^{\alpha} q^{d}\right\} \mathrm{d} x \\
\leq C\left(\left\|\operatorname{div} q^{d}\right\|_{L^{\infty}}\left\|\nabla^{\alpha}\left(\frac{1}{\rho^{\tau} e_{\theta}^{\tau}}\right)\right\|\left\|\nabla^{\alpha} \theta^{d}\right\|+\left\|\left(\nabla\left(\frac{1}{\rho^{\tau} e_{\theta}^{\tau}}\right), \nabla\left(\frac{1}{\theta^{\tau}}\right)\right)\right\|_{L^{\infty}}\left\|\nabla^{\alpha} q^{d}\right\|\left\|\nabla^{\alpha} \theta^{d}\right\|\right) \\
\leq \varepsilon\left\|\nabla^{\alpha} q^{d}\right\|^{2}+C\left(\left(E^{d}\right)^{2}+\tau\left(E^{d}\right)^{3}+\tau^{2}\left(E^{d}\right)^{4}\right), \\
G_{3} \leq C\left\|\nabla^{\alpha}\left(u^{\tau} \nabla u^{d}\right)\right\|\left\|\nabla^{\alpha} u^{d}\right\| \\
\leq C\left(\left\|u^{\tau}\right\|_{L^{\infty}}\left\|\nabla^{\alpha+1} u^{d}\right\|+\left\|\nabla u^{d}\right\|_{L^{\infty}}\left\|\nabla^{\alpha} u^{\tau}\right\|\right)\left\|\nabla^{\alpha} u^{d}\right\| \\
\leq \varepsilon\left\|\nabla^{\alpha+1} u^{d}\right\|^{2}+C(\varepsilon)\left(\left(E^{d}\right)^{2}+\tau\left(E^{d}\right)^{3}\right)
\end{gathered}
$$

and

$$
\begin{aligned}
\left|T_{i}\right| & \leq\left\|\left(\rho_{t}^{\tau}, \theta_{t}^{\tau}\right)\right\|_{L^{\infty}}\left(E^{d}\right)^{2} \leq C\left(1+\tau\left\|\left(\rho_{t}^{d}, \theta_{t}^{d}\right)\right\|_{L^{\infty}}\right)\left(E^{d}\right)^{2} \\
& \leq C\left(1+\tau\left(E^{d}+\tau\left(E^{d}\right)^{2}+\left\|q^{d}\right\|_{s}\right)\right)\left(E^{d}\right)^{2} \\
& \leq C\left(\left(E^{d}\right)^{2}+\tau\left(E^{d}\right)^{3}+\tau^{2}\left(E^{d}\right)^{4}\right)+\varepsilon\left\|q^{d}\right\|_{s}^{2} .
\end{aligned}
$$

By choosing $\varepsilon$ sufficiently small and using assumption A.2, we conclude that (3.22) holds and this completes the proof. 
Using Lemma 3.4, we can show that $E^{d}$ is uniformly bounded. In fact, from Lemma 3.4, we derive that

$$
\frac{\mathrm{d}}{\mathrm{d} t} g \leq \widehat{C}\left(1+g+\tau^{2} g^{2}\right)
$$

for some $\widehat{C}>0$, where $g:=\left(E^{d}\right)^{2}$. We assume a priori that

$$
g \leq e^{2 \widehat{C} T}-1
$$

We will show that $g \leq \frac{1}{2}\left(e^{2 \widehat{C} T}-1\right)$ holds if we choose $\tau$ small enough such that $\tau^{2} \leq \frac{1}{e^{2 \widehat{C} T}-1}$. This justifies the a priori estimate (3.25) and thus proves our result. In fact, if $\tau^{2} \leq \frac{1}{e^{2 \widehat{C} T}-1}$, we get $g \leq \frac{1}{\tau^{2}}$ and thus $\tau^{2} g^{2} \leq g$. Hence, the inequality (3.24) turns into

$$
\frac{\mathrm{d}}{\mathrm{d} t} g \leq \widehat{C}(1+2 g)
$$

Solving the above inequality, we immediately get $g \leq \frac{1}{2}\left(e^{2 \widehat{C} T}-1\right)$. This finishes the proof of the main Theorem 3.1.

\section{REFERENCES}

[1] Y. Cho and B.J. Jin, Blow-up of viscous heat-conducting compressible flows, J. Math. Anal. Appl. 320 (2) (2006), 819-826.

[2] H.J. Choe and H. Kim, Strong solutions of the Navier-Stokes equations for isentropic compressible fluids, J. Differ. Eqs. 190 (2003), 504-523.

[3] D. Hoff, Global existence for 1D, compressible, isentropic Navier-Stokes equations with large initial data, Trans. Amer. Math. Soc. 303 (1) (1987), 169-181.

[4] D. Hoff, Global solutions of the Navier-Stokes equations for multidimensional compressible flow with discontinuous initial data, J. Differ. Eqs. 120 (1) (1995), 215-254.

[5] H.D. Fernández Sare and R. Racke, On the stability of damped Timoshenko systems Cattaneo versus Fourier law, Arch. Rational Anal. Mech. 194 (2009), 221-251.

[6] E. Feireisl, A. Novotny and H. Petzeltová, On the existence of globally defined weak solutions to the Navier-Stokes equations, J. Math. Fluid Mech. 3 (2001), 358-392.

[7] Y. Hu and R. Racke, Formation of singularities in one-dimensional thermoelasticity with second sound, Quart. Appl. Math. 72 (2014), 311-321.

[8] X.D. Huang, J. Li and Z.P. Xin, Global well-posedness of classical solutions with large oscillations and vacuum to the three-dimensional isentropic compressible Navier-Stokes equations, Comm. Pure. Appl. Math. 65 (2012), 549-585.

[9] S. Jiang, On the asymptotic behavior of the motion of a viscous, heat-conducting, onedimensional real gas, Math. Z. 216 (1994), 317-336.

[10] S. Jiang and R. Racke, Evolution equations in thermoelasticity. $\pi$ Monographs Surveys Pure Appl. Math. 112. Chapman \& Hall/CRC, Boca Raton (2000).

[11] S. Jiang and P. Zhang, Global spherically symmetry solutions of the compressible isentropic Navier-Stokes equations, Comm. Math. Phys. 215 (2001), 559-581.

[12] S. Jiang and P. Zhang, Axisymmetric solutions of the 3-D Navier-Stokes equations for compressible isentropic fluids, J. Math. Pures. Appl. 82 (2003), 949-973.

[13] S. Kawashima, Systems of a hyperbolic-parabolic composite type, with applications to the equations of magnetohydrodynamics, Thesis, Kyoto University (1983). 
[14] P.L. Lions, Mathematical Topics in Fluid Mechanics, Vol.I, Incompressible Models. Clarendon Press, Oxford (1996).

[15] P.L. Lions, Mathematical Topics in Fluid Mechanics, Vol.II, Compressible Models. Clarendon Press, Oxford (1998).

[16] A. Matsumura and T. Nishida, The initial value problem for the equations of motion of viscous and heat-conductive gases, J. Math. Kyoto Univ. 20 (1) (1980), 67-104.

[17] A. Matsumura and T. Nishida, Initial Boundary Value Problems for the Equations of Motion of Compressible Viscous and Heat-Conductive Fluids, Comm. Math. Phys. 89 (1983), 445-464.

[18] J. Nash, Le problème de Cauchy pour les équations différentielles dún fluide général, Bull. Soc. Math. France 90 (1962), 487-497.

[19] R. Racke, Thermoelasticity with second sound - exponential stability in linear and nonlinear 1-d, Math. Methods Appl. Sci. 25 (2002), 409-441.

[20] R. Racke, Thermoelasticity. Chapter 4 in: Handbook of Differential Equations. Vol. 5. Evolutionary Equations. Eds.: C.M. Dafermos, M. Pokorný. Elsevier (2009), 315-420.

[21] J. Serrin, On the uniqueness of compressible fluid motion, Arch. Rational Mech. Anal. 3 (1959), 271-288.

[22] Y. Shizuta and S. Kawashima, Systems of equations of hyperbolic-parabolic type with applications to the discrete Boltzmann equation. Hokkaido Math. J. 14 (1985), 249-275.

[23] Z.P. Xin, Blowup of smooth solutions to the compressible Navier-Stokes equation with compact density, Comm. Pure. Appl. Math. 51 (1998), 229-240.

[24] W. Yong, A note on the zero mach number limit of compressible euler equations, P. AMS, 133 (2005), 3079-3085.

Yuxi Hu, LCP, Institute of Applied Physics and Computational Mathematics, Beijing 100088, P.R. China, yxhu86@163.com

Reinhard Racke, Department of Mathematics and Statistics, University of Konstanz, 78457 Konstanz, Germany, reinhard.racke@uni-konstanz.de 